\section{Drug-loaded biodegradable microspheres for image-guided combinatory epigenetic therapy in cells}

\author{
Ronald X. Xu, ${ }^{a, *}$ Jeff S. Xu, ${ }^{a, *}$ Tao Zuo, ${ }^{b}$ Rulong Shen, ${ }^{c}$ \\ Tim H. Huang, ${ }^{b}$ and Michael F. Tweedle ${ }^{d}$ \\ aThe Ohio State University, Department of Biomedical Engineering, \\ Columbus, Ohio 43210 \\ bThe Ohio State University, Department of Molecular Virology, \\ Immunology and Medical Genetics, Columbus, Ohio 43210 \\ 'The Ohio State University, Department of Pathology, Columbus, \\ Ohio 43210 \\ dThe Ohio State University, Department of Radiology, Columbus, \\ Ohio 43210
}

\begin{abstract}
We synthesize drug-loaded poly (lactic-coglycolic acid) (PLGA) microspheres for image-guided combinatory epigenetic therapy in MCF-10A human mammary epithelial cells. LY294002 and Nile Red are encapsulated in microspheres for sustained drug release and fluorescence microscopic imaging. Drug-loaded microspheres target MCF-10A cells through a three-step binding process involving biotinylated antibody, streptavidin, and biotinylated microspheres. LY294002 loaded microspheres and 5-Aza2-deoxycytidine are applied to MCF-10A cells for combinatory PI3K/AKT inhibition and deoxyribonucleic acid (DNA) demethylation. Our study implies the technical potential of disease targeting and image-guided combinatory epigenetic therapy using drug-loaded multifunctional biodegradable PLGA microspheres. $\odot 2011$ Society of Photo-Optical Instrumentation Engineers (SPIE). [DOI: 10.1117/1.3548878]
\end{abstract}

Keywords: poly (lactic-co-glycolic acid) (PLGA); PI3K/AKT inhibition; DNA demethylation; microsphere; epigenetic therapy; biotinylation; fluorescence imaging.

Paper 10545LR received Oct. 6, 2010; revised manuscript received Jan. 6, 2011; accepted for publication Jan. 7, 2011; published online Feb. 16, 2011.

Recent research in epigenetic alterations opens a new avenue for cancer detection, prognosis, and treatment. ${ }^{1}$ Epigenetic events are associated with mitotically heritable changes in phenotype or gene expression that do not affect the primary DNA sequence. Since many processes of tumor development and progression are mediated by epigenetic events, targeting epigenetic changes becomes an appealing strategy for cancer treatment. A number of DNA demethylation reagents and histone-deacetylase inhibitors have been tested in preclinical studies and clinical trials. ${ }^{2,3}$ Combinatory therapies involving these inhibitors have shown synergistic anti-cancer effects. ${ }^{4}$ It has also been demonstrated that the activation of PI3K/AKT oncogenic signaling

*The first two authors contribued equally to this work. For questions on microfabrication: xu.202@osu.edu; for questions on epigenetic therapy; tim.huang@osumc.edu; for questions on pharmaceutics: michael.tweedle@ osumc.edu.

Address all correspondence to: Ronald Xu, The Ohio State University, Biomedical Engineering, 270 Bevis Hall, 1080 Carmack Rd., Columbus, Ohio 43210. Tel: 614 6883635; Fax: 614 2927309; E-mail: xu.202@osu.edu. triggers the epigenetic silencing in mammary epithelial cells, suggesting the clinical potential of PI3K/AKT inhibition and DNA demethylation in combinatory epigenetic therapy. 5,6

One of the key success factors for combinatory epigenetic therapy is the introduction of long term epigenetic modifications without acute cytotoxicity. However, existing drug delivery methods do not facilitate sustained and controlled simultaneous release of several drugs in low doses. Without dose control, combinatory therapies will have a limited synergistic effect because of variations in solubility, toxicity, and release kinetics of individual drug components. Image-guidance is also important in combinatory therapies because it will provide real-time guidance of disease targeting and feed back control of drug concentration. We have previously synthesized poly (lactic-co-glycolic acid) (PLGA) microbubbles and nanoparticles for biomedical imaging and therapeutic applications. ${ }^{7-10}$ These microbubbles and nanoparticles can be potentially used as a multifunctional biodegradable carrier for cancer targeting and image-guided epigenetic therapy. In this letter, we demonstrate the technical potential of image-guided combinatory epigenetic therapy in cells using drug-loaded PLGA microspheres. LY294002, a PI3K/AKT inhibitor, was encapsulated in PLGA microspheres for sustained drug release. The surface of PLGA microspheres were modified to target the epithelial specific antigen (ESA) expressed on the cell surface of MCF-10A human mammary epithelial cells. The microsphere-assisted combinatory PI3K/AKT inhibition and DNA demethylation was also demonstrated. To the best of the authors' knowledge, this research is the first attempt to fabricate drug-loaded microspheres for image-guided combinatory epigenetic therapy.

Synthesis of drug-loaded microspheres. A micro-emulsion method was used to encapsulate Nile Red dye (Sigma, St. Louis, Missouri) and LY294002 (Cayman Chemical, Missouri) in carboxylic acid group terminated PLGA (50/50, MW 12000, RG 502H, Boehringer Ingelheim, Germany) microspheres.

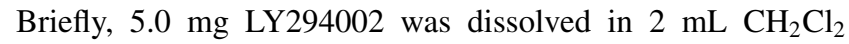
and then added $44.7 \mathrm{mg}$ PLGA and Nile Red with a final concentration of $2 \mu \mathrm{g} / \mathrm{mL}$. The solution was mixed with $10 \mathrm{~mL}$ polyvinyl alcohol ( $3 \mathrm{w} / \mathrm{v} \%)$ and emulsified at $8000 \mathrm{rpm}$ for $3 \mathrm{~min}$. The emulsion was then added to $30 \mathrm{~mL}$ isopropanol solution $(5 \mathrm{v} / \mathrm{v} \%)$, stirred for $3 \mathrm{~h}$, centrifuged at $2000 \mathrm{rpm}$ for $7 \mathrm{~min}$, washed 3 times with deionized water, and freeze-dried in the dark for $24 \mathrm{~h}$. Figure 1(a) shows the scanning electron microscopic (SEM) image of the fabricated microspheres. The averaged size of the microspheres was $1.34 \pm 0.94 \mu \mathrm{m}$, as characterized by a BI-200SM Dynamic Laser Scattering System (Particle Sizing Systems Inc., California). The drug encapsulating rate was $2.1 \%$, as characterized by an Agilent UV-VIS spectrophotometer (Agilent Technologies, California).

Characterization of drug release profile. Drug-loaded microspheres $(15.7 \mathrm{mg})$ were suspended in a release medium of $25 \mathrm{~mL}$ phosphate buffered saline (PBS) buffer $(p \mathrm{H}=7.4)$. The medium was sealed in an amber glass bottle and gently stirred at $300 \mathrm{rpm}$ at room temperature. At the selected time points, $2 \mathrm{~mL}$ medium sample was drawn and centrifuged at $10,000 \mathrm{rpm}$ for $3 \mathrm{~min}$. The supernatant was collected for ultraviolet-visible (UV-Vis) measurement of drug concentration. The measurement was

1083-3668/2011/16(2)/020507/3/\$25.00 (C) 2011 SPIE 


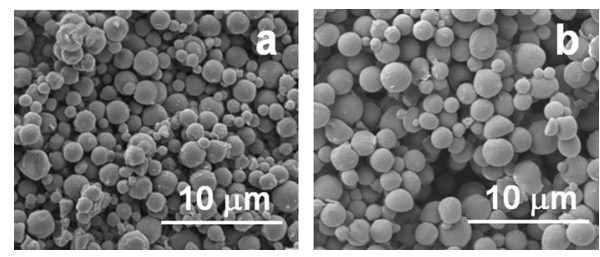

Fig. 1 SEM image of drug-loaded microspheres: (a) before the release test, (b) after the six day release test.

taken in triplicate. After each measurement, the medium sample was resuspended and returned to the bottle. At the end of the experiment, the sample was centrifuged and washed. Drug-loaded microspheres were collected, freezing-dried, and kept at $4^{\circ} \mathrm{C}$ for further SEM imaging. As the control, an equivalent amount of pure LY294002 was dissolved in $25 \mathrm{~mL}$ PBS buffer $(p \mathrm{H}=7.4)$. The drug release profiles for both the sample and the control were monitored for six days. As can be seen from Fig. 2, pure LY294002 reaches the saturated concentration immediately after it suspends in PBS, whereas drug-loaded microspheres release LY294002 slowly. Figure 1(b) shows the SEM image of drug-loaded microspheres after the six day release test. Compared with Fig. 1(a), the shape and the dimension of microspheres do not change significantly, indicating the possible drug release mechanism dominated by diffusion and dissolution.

Targeted binding and microscopic imaging. PLGA microspheres were N-Hydroxysuccinimide ester activated and biotyinylated following our previous protocol. ${ }^{8}$ The targeted binding of these microspheres was demonstrated in MCF-10A human mammary epithelial cells incubated in Lab-Tek ${ }^{\mathrm{TM}}$ chambers (Sigma, St. Louis, Missouri) in a humidified atmosphere of $5 \% \mathrm{CO}_{2}$ at $37^{\circ} \mathrm{C}$. Before the binding test, the cell monolayer was washed with PBS, fixed with $3.7 \%$ paraformaldehyde, and blocked nonspecific binding by $2 \%$ bovine serum albumin (Sigma, St. Louis, Missouri). The binding process consists of three consecutive steps. First, $0.5 \mathrm{nmol}$ biotinylated antibody,

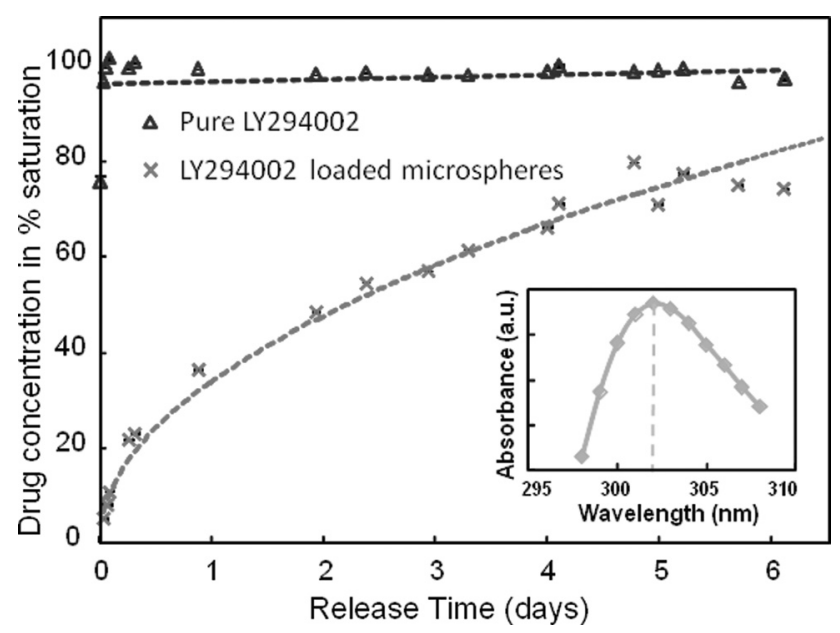

Fig. 2 LY294002 release profile from drug-loaded microspheres versus that of pure LY294002 without microsphere encapsulation. The drug concentration is derived from the UV-VIS spectrum of LY294002, as shown in the inset. Drug-loaded microspheres facilitate slow release of LY294002, while pure LY294002 without microsphere encapsulation reaches the saturated concentration immediately.

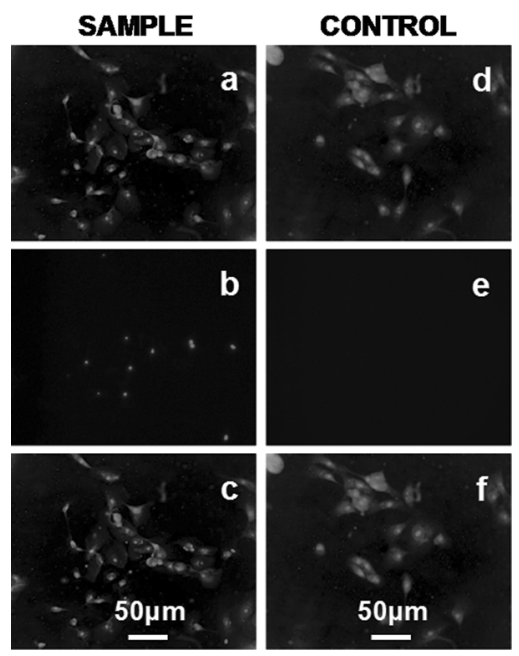

Fig. 3 MCF-10A cells after applying the biotinylated microspheres following a three-step binding process (left images, sample) and after applying the bare microspheres without biotinylation (right images, control). (a,d) Microscopic images of the stained MCF-10A cells. (b,e) Fluorescence images showing the bounded microspheres. $(\mathrm{C}, \mathrm{f})$ Fusions of microscopic and fluorescence images.

ESA [B29.1(VU-ID9)] (Biotin) antibody, (GenWay BioTech, California) was applied to the cultured MCF-10A cells to target the over-expressed ESA. The cells were then incubated for $1 \mathrm{~h}$ and washed 3 times with PBS buffer. Secondly, 0.5 nmol streptavidin was applied and the cells were incubated for $0.5 \mathrm{~h}$ and washed 3 times with PBS. Finally, the biotinylated microspheres were applied. The cells were incubated for $0.5 \mathrm{~h}$ and washed 3 times with PBS. After the above process, the cells were stained with Diff-Quik (Fisher Scientific, Pittsburgh, Pennsylvania) for three dips, washed in tap water for $1 \mathrm{~s}$, air-dried and coverslipped with Richard-Allan Scientific Mounting Medium (Kelanmazoo, Missouri) for microscopic and fluorescence imaging. In comparison, bare microspheres without biotinylation were also applied to MCF-10A cells. As can be seen in Fig. 3, the biotinylated microspheres bind with the cells, while bare microspheres do not bind with the cells. The binding efficiency as shown in Fig. 3 does not reflect the in vivo situation since the sample has been washed several times before fluorescence imaging. In addition, the size of the microspheres and the ESA expression of MCF-10A cells also affect the binding of the microspheres. For future therapeutic applications, the binding efficiency can be enhanced by optimizing the particle size, by improving the surface conjugation efficiency, and by using multivalent targeting strategies.

Microsphere-assisted combinatory epigenetic therapy. We have previously reported the combinatory therapeutic effect of LY294002 (for PI3K/AKT inhibition) and DAC (for DNA demethylation). ${ }^{6}$ In this paper, we further demonstrated the use of drug-loaded microspheres for the combinatory epigenetic therapy. The following therapeutic agents were prepared in advance: 1. PBS suspension of LY294002 loaded PLGA microspheres, 2. PBS solution of DAC, 3. PBS solution of LY294002, and 4. PBS suspension of bare PLGA microspheres with no drug loaded. Four sets of Petri dishes were prepared (3 dishes for each set). $5000 \mathrm{MCF}-10 \mathrm{~A}$ cells were transferred to each of the Petri dishes and incubated in medium for 2 days. After 


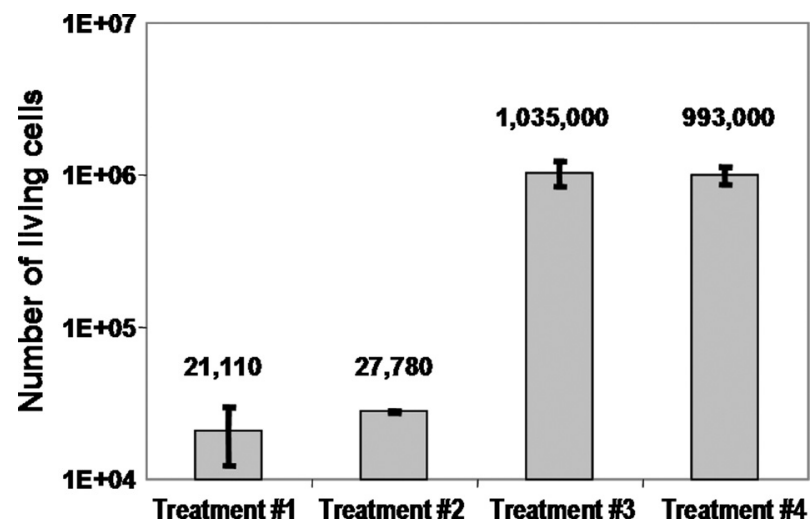

Fig. 4 Number of living cells after the following treatments: (1) LY294002 loaded PLGA microspheres and DAC, (2) LY294002 and DAC, (3) bare microspheres, and (4) blank control. Similar to the combinatory LY294402 and DAC therapy, applying LY294002 loaded microspheres and DAC significantly inhibits the proliferation of MCF-10A cells. In comparison, bare PLGA microspheres do not affect the cell proliferation.

that, the following treatments were applied to each set of Petri dishes respectively: 1. agents \#1 and \#2 (equivalent LY294002 dose: $5 \mu \mathrm{M}$, DAC dose: $1 \mu \mathrm{M}$ ), 2. agents \#2 and \#3 (equivalent LY294002 dose: $5 \mu \mathrm{M}$, DAC dose: $1 \mu \mathrm{M})$, 3. agent \#4 (microsphere concentration equivalent to that of agent \#1), and 4. blank control medium. The cells were treated for 4 days with medium changed everyday, followed by incubation in medium for 3 more days. By the end of the experiments, the cells in each dish were collected and counted by a Countess ${ }^{\mathrm{TM}}$ Automated Cell Counter (Invitrogen, Carlsbad, California) and a haemocytometer (Sigma, St. Louis, Missori). As can be seen in Fig. 4, applying LY294002-loaded microspheres and DAC significantly inhibits the cell proliferation, similar to that of pure LY294002 and DAC. In comparison, applying bare microspheres with no drug loaded does not affect the cell proliferation, similar to that of the blank control. These results suggest that PLGA microspheres can be used as a biocompatible carrier for combinatory epigenetic therapy. Furthermore, Fig. 4 shows stronger inhibition of cell proliferation for LY294002-loaded microspheres than LY294002. However, this observation does not have statistical power due to the small sampling size $(n=3)$, the large deviation of cell numbers, and the short treatment time. Further evaluation of the long-term therapeutic effect requires more extensive experiments.

In summary, this letter reports our first attempt to fabricate and conjugate drug-loaded microspheres for image-guided combinatory epigenetic therapy in MCF-10A cells. Although MCF$10 \mathrm{~A}$ is not a cancer cell line, it is commonly used to explore new imaging and drug delivery regimes in breast cancer studies. In the future, we will test microsphere-assisted epigenetic ther- apies in cancer cell lines and cancer xenograft models. We will also encapsulate multiple epigenetic drugs in PLGA carriers and optimize the release profiles of individual drug components for maximal anti-cancer synergy. In addition to cancer therapy, the technique described in this letter will provide a general platform for image-guided synergistic therapy of many other diseases and tissue anomalies.

\section{Acknowledgments}

This work was supported in part by National Institutes of Health (U54 CA113001 to T.H.H., R01 CA 069065 to T.H.H.) Department of Defense Breast Cancer Research Program (W81XWH07 to R.X.X.), and OSU Department of Radiology (startup fund to M.F.T.). The authors are grateful to Dr. Haiming Ding of Department of Radiology for his technical input and Mr. Jiwei Huang of Department of Biomedical Engineering for his image processing assistance.

\section{References}

1. C. B. Yoo and P. A. Jones, "Epigenetic therapy of cancer: past, present and future," Nat. Rev. Drug Discovery 5(1), 37-50 (2006).

2. J. E. Bolden, M. J. Peart, and R. W. Johnstone, "Anticancer activities of histone deacetylase inhibitors," Nat. Rev. Drug Discovery 5(9), 769-784 (2006).

3. J. P. Issa and H. M. Kantarjian, "Targeting DNA methylation," Clin. Cancer Res. 15(12), 3938-3946 (2009).

4. E. E. Cameron, K. E. Bachman, S. Myohanen, J. G. Herman, and S. B. Baylin, "Synergy of demethylation and histone deacetylase inhibition in the re-expression of genes silenced in cancer," Nat. Genet. 21(1), 103-107 (1999)

5. H. J. Lin, T. Zuo, C. H. Lin, C. T. Kuo, S. Liyanarachchi, S. Sun, R. Shen, D. E. Deatherage, D. Potter, L. Asamoto, S. Lin, P. S. Yan, A. L. Cheng, M. C. Ostrowski, and T. H. Huang, "Breast cancer-associated fibroblasts confer AKT1-mediated epigenetic silencing of Cystatin M in epithelial cells," Cancer Res. 68(24), 10257-10266 (2008).

6. T. Zuo, T.-M. Liu, C. Taslim, X. Lan, B. Kennedy, D. E. Deatherage, Y.-I. Weng, S. Liyanarachchi, P.-Y. Hsu, J. C. Liu, S. Lin, K. Huang, H.-J. L. Lin, A. S. L. Cheng, V. X. Jin, and T. Huang, "Epigenetic Silencing Mediated Through Activated PI3K/AKT Signaling in Breast Cancer," Cancer Res. (in press 2011).

7. R. Xu, J. Huang, J. Xu, D. Sun, G. Hinkle, E. W. Martin, and S. Povoski, "Fabrication of indocyanine green encapsulated biodegradable microbubbles for structural and functional imaging of cancer," J. Biomed. Opt. 14(3), 034020 (2009).

8. J. S. Xu, J. Huang, R. Qin, G. H. Hinkle, S. P. Povoski, E. W. Martin, and $\mathrm{R}$. X. Xu, "Synthesizing and binding dual-mode poly (lactic-coglycolic acid) (PLGA) nanobubbles for cancer targeting and imaging," Biomaterials 31(7), 1716-1722 (2010).

9. L. Zhang, J. S. Xu, V. M. Sanders, A. D. Letson, C. J. Roberts, and R. X. Xu, "Multifunctional microbubbles for image-guided antivascular endothelial growth factor therapy," J. Biomed. Opt. 15(3), 030515 (2010).

10. C. Kim, R. Qin, J. S. Xu, L. Wang, and R. X. Xu, "Ultrasound and photoacoustic dual-modal imaging of thick biological tissue with microbubble enhancement," J. Biomed. Opt. 15(1), 010510 (2010). 